This is the accepted manuscript made available via CHORUS, the article has been published as:

\title{
Plasmonic enhancement of nonlinear magneto-optical response in nickel nanorod metamaterials
}

V. L. Krutyanskiy, I. A. Kolmychek, E. A. Gan'shina, T. V. Murzina, P. Evans, R. Pollard, A. A.

Stashkevich, G. A. Wurtz, and A. V. Zayats

Phys. Rev. B 87, 035116 - Published 11 January 2013

DOI: 10.1103/PhysRevB.87.035116 


\title{
Plasmonic-enhancement of magnetic nonlinear-optical response in nickel nanorod metamaterial
}

\author{
V.L. Krutyanskiy ${ }^{1}$, I.A. Kolmychek ${ }^{1}$, E.A. Gan'shina ${ }^{1}$, T.V. Murzina ${ }^{1}$, P. \\ Evans $^{2}$, R. Pollard ${ }^{2}$, A. A. Stashkevich ${ }^{3}$, G.A. Wurtz ${ }^{4}$ and A. V. Zayats ${ }^{4}$ \\ ${ }^{1}$ Department of Physics, Moscow State University, 119991, Moscow, Russia \\ ${ }^{2}$ Centre for Nanostructured Media, IRCEP, The Queen's University of Belfast, Belfast, BTr 1NN, United Kingdom \\ ${ }^{3}$ LSPM CNRS (UPR 3407), Université Paris 13, \\ Sorbonne Paris Cité, 93430 Villetaneuse, France and \\ ${ }^{4}$ Physics Department, King's College London, Strand, London WC2R 2LS, United Kingdom
}

(Dated: December 28, 2012)

\begin{abstract}
Linear and nonlinear magneto-optical properties of the metamaterials based on nickel nanorod assemblies have been studied. The second-harmonic generation properties follow the effective medium description of the metamaterial as a homogeneous layer isotropic in the direction normal to the nanorod axes. Magneto-optical and nonlinear magneto-optical Kerr effects are enhanced in the vicinity of the plasmonic modes of $\mathrm{Ni}$ nanorods and of the photonic resonance of the metamaterial layer, while conventional (nonmagnetic) optical response does not exhibit plasmonic-related features. The observed surprisingly high sensitivity of magneto-optical and nonlinear magneto-optical responses to the metamaterial photonic modes is prospective for the diagnostics of plasmonic excitations in magnetic nanostructures and for possible applications in high density magneto-optical data storage.
\end{abstract}

PACS numbers: 
Magnetic plasmonic nanostructures allow for a dramatic improvement in the efficiency of magneto-optical (MO) interactions making them promising materials for applications in high-density information storage, signal conditioning and information processing ${ }^{1-3}$. Ferromagnetic metals can support surface plasmon excitations in certain wavelength ranges where the real part of the permittivity is negative $(\operatorname{Re} \epsilon<0)$, allowing the excitation of collective electron oscillations near the surface. Plasmonic effects in optical and magneto-optical behaviour have been reported in the case of purely ferromagnetic metal nanostructures as well as in hybrid ferromagnetic-noble-metal composites ${ }^{4-9}$. Arrays of micron-long parallel Ni nanowires have been seen to exhibit strongly enhanced MO activity compared to bulk $\mathrm{Ni}^{5}$. Numerical simulations based on the scattering matrix method indicate that the origin of this enhancement is associated with the plasmonic excitations along individual nanowires ${ }^{6}$. Multilayered thin films and core-shell nanoparticles of noble metals and ferromagnetic materials ${ }^{7,8,10-12}$ have also been used to engineer enhancement of magneto-optical properties via resonant plasmonic excitations. $\mathrm{Ni}$ and Co nanorod-based metamaterials have recently been studied showing enhanced magnetic response ${ }^{13}$ and asymmetry of magneto-optical interactions in Brilloiun scattering experiments ${ }^{14,15}$.

At the same time, optical second-harmonic generation (SHG) is known to be an extremely sensitive probe of surfaces, interfaces and nanostructures ${ }^{16-20}$. Moreover, it has been shown that the nonlinear MO Kerr and Faraday effects that appear under SHG excitation are orders of magnitude larger than linear-optical ones. Combining the field-enhancement effects provided by plasmonic excitations with a strong sensitivity to the magnetic state of the material, nonlinear magneto-optical Kerr-effects may be instrumental in studying weak surface plasmon resonances in magnetic materials and in the development of new applications.

In this paper, we present a comprehensive study of the magneto-optical and nonlinear magneto-optical response of a metamaterial consisting of closely-spaced nickel nanorods. The effective medium model of the metamaterial as an in-plane isotropic layer is used for the description of the linear optical properties and second-harmonic generation. We show that plasmonic excitations can be observed in both the linear and nonlinear MO response, while no plasmonic features are observed in the non-magnetic response. The observed surprisingly high sensitivity of magneto-optical responses to the plasmonic properties of metamaterials opens up new opportunities for studying plasmonic behaviour in magnetic nanostructures and applications in high density data storage. The tailoring metamaterial effective permittivity with the nanorod geometrical parameters allows engineering plasmonic and photonic metamaterial modes to further control spectral response of the enhanced magneto-optical effects.

The magnetic metamaterial has been fabricated via templated electrodeposition of $\mathrm{Ni}^{13}$. Initially, an aluminum film was sputtered on a silicon substrate with buffer $\mathrm{Au}$ and $\mathrm{Ta}_{2} \mathrm{O}_{5}$ layers. The nanopores were formed perpendicular to the $\mathrm{Al}$ film surface during anodization of the film in sulfuric acid. The pores were filled with nickel by means of $\mathrm{NiSO}_{4}$ electrolysis ${ }^{14}$. The obtained nanorods of diameter around $20 \mathrm{~nm}$ and length $175 \mathrm{~nm}$ form a quasi-periodic hexagonal lattice with the average distance between the rods being about $40 \mathrm{~nm}$. Magnetization measurements have indicated that the structure under investigation is ferromagnetic with an in-plane magnetization saturation magnetic field of about $1 \mathrm{kOe}$.

The transverse MO Kerr effect (magnetization lying in the sample plane and perpendicular to the incidence light plane) was studied using $p$-polarized light ${ }^{21}$. The angle of incidence was $68^{\circ}$ and the wavelength was varied in the range from $300 \mathrm{~nm}$ to $850 \mathrm{~nm}$. As a measure of the transverse magneto-optical Kerr effect (TKE), the TKE contrast is commonly used, defined as $\rho_{\omega}=\frac{I_{\omega}(M)-I_{\omega}(-M)}{I_{\omega}(M)+I_{\omega}(-M)}$, where $I_{\omega}(M)$ and $I_{\omega}(-M)$ are the intensities of the linearly polarised reflected light for the opposite directions of the in-plane applied DC magnetic field of $\mathrm{M}=3 \mathrm{kOe}$, respectively.

For the nonlinear optical experiments, the output of a Ti:sapphire laser was used in the spectral range 730-830 $\mathrm{nm}$ with the pulse duration of about $80 \mathrm{fs}$, repetition rate of $80 \mathrm{MHz}$ and a mean power of $150 \mathrm{~mW}$. The pump radiation was focused on the sample into a spot of about $50 \mu \mathrm{m}$ in diameter. SHG radiation in the direction of the specular reflection was spectrally selected by colour filters, passed through the analyzer and detected by a photomultiplier operating in the photon counting mode. Magnetization-induced effects in SHG were studied in the geometry of the transverse magneto-optical Kerr effect. To characterize the magnetization-induced changes in the SHG intensity, its magnetic contrast, defined similar to the linear case as $\rho_{2 \omega}=\frac{I_{2 \omega}(M)-I_{2 \omega}(-M)}{I_{2 \omega}(M)+I_{2 \omega}(-M)}$ was used ${ }^{9}$, where $I_{2 \omega}(M)$ and $I_{2 \omega}(-M)$ are the SHG intensities measured for the opposite directions of the magnetization $\vec{M}$. Here, $I_{2 \omega}(M) \propto\left[\vec{E}_{2 \omega}^{c r}+\vec{E}_{2 \omega}^{\text {magn }}(M)\right]^{2}$, $I_{2 \omega}(-M) \propto\left[\vec{E}_{2 \omega}^{c r}+\vec{E}_{2 \omega}^{m a g n}(-M)\right]^{2}$, and $\vec{E}_{2 \omega}^{c r}$ and $\vec{E}_{2 \omega}^{\text {magn }}(M)$ are the SH fields related to crystallographic, independent of the magnetization, and magnetization, depending on $\vec{M}$, contributions, respectively.

The linear reflection spectra of the metamaterial are shown in Fig. 1(b) for $45^{\circ}$ and $70^{\circ}$ angles of incidence. It can be seen that for a particular case of the s-polarized light the spectrum blue-shifts to several dozens of nanometers when the angle of incidence is increased. The spectra can be understood in terms of the multiple interference in an anisotropic metamaterial slab formed by the Ni nanorods in an alumina matrix. This is confirmed by effective medium modelling ${ }^{22}$ presented in Fig. 1 (c) for $70^{\circ}$ angle of incidence and when taking into account the parameters of the nanorods in this spectral range ${ }^{25}$. The difference in the effective permittivity parameters $\varepsilon_{x}$ and $\varepsilon_{z}$ in Fig. 1 (d) indicates a strongly anisotropic behaviour of the metamaterial with high values of loss; here, $\mathrm{z}$ is the direction normal 
to the metamaterial film and parallel to the nanorods' axes. In the spectral range below $800 \mathrm{~nm}$, the metamaterial does not exhibit hyperbolic dispersion, and both $\varepsilon_{x}$ and $\varepsilon_{z}$ are positive as a consequence of the low filling factor of $\mathrm{Ni}$ in the host matrix. It can be inferred from the simulations that strong Ohmic losses in the Ni nanorods lead to a very weak interaction between the nanorod plasmonic excitations. It should be noted that for a single Ni nanorod embedded in an $\mathrm{Al}_{2} \mathrm{O}_{3}$ matrix, the localized surface plasmon (LSP) resonance conditions are satisfied at approximately $380 \mathrm{~nm}$ and $2000 \mathrm{~nm}$ for the electromagnetic field polarization perpendicular and parallel to the nanorod axis, respectively.
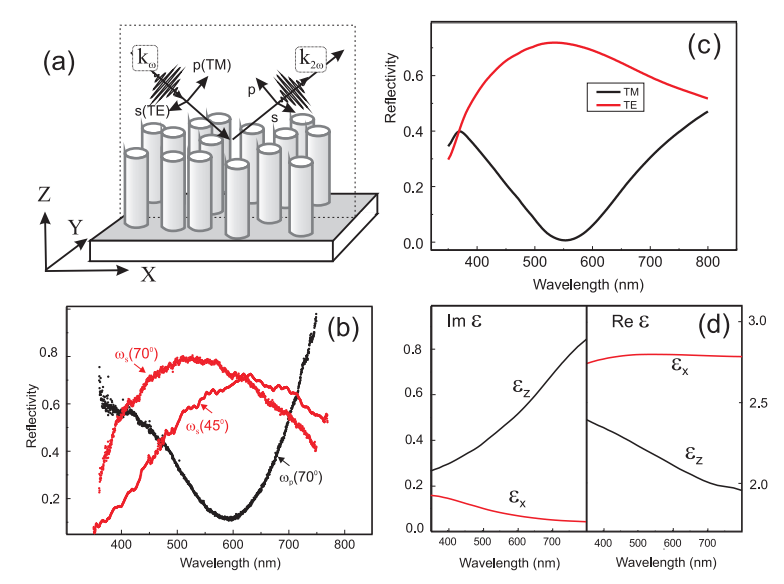

FIG. 1. (a) Schematic view of the sample of the Ni nanorod metamaterial, geometry of light interaction and the coordinate system. (b) Experimental reflectivity spectra measured at $45^{\circ}$ and $70^{\circ}$ angles of incidence and (c) effective-medium-modelled reflectivity spectra of the metamaterial on a Si-substrate at $70^{\circ}$ angle of incidence. (d) Real and imaginary parts of the effective permittivity of the anisotropic metamaterial calculated in the Maxwell-Garnet approximation.

The spectrum of the linear TKE contrast in the metamaterial (Fig. 2) reveals a clear maximum at around 380 $\mathrm{nm}$ that is close to the LSP resonance of the Ni nanorods associated with the electron motion perpendicular to the nanorod axis ${ }^{5}$. The wide MO peak observed at around $600 \mathrm{~nm}$ corresponds to the Fabry-Perot resonance-related p-polarized photonic mode of the anisotropic metamaterial layer for this angle of incidence (cf. Fig. 1 (b,c)). This mode is determined by the effective optical parameters of the layer and its thickness. It should be noted that far from these resonances (both LSP resonance and photonic mode of the metamaterial layer), the value of the magneto-optical Kerr effect is relatively small. This is probably because under nonresonant conditions, thin composite films with a small volume fraction of Ni do not possess strong magneto-optical activity. Since the MO contrast corresponds to the ratio of the magnetic and nonmagnetic components of the effective dielectric constant, it has a maximum at around $600 \mathrm{~nm}$, where the spectrum of the reflected radiation has a minimum due to the interference in the anisotropic metamaterial layer. At the same time, a smooth Ni film of the same thickness exhibits weaker, despite larger amount of $\mathrm{Ni}$, magnetic contrast with different spectral response (Fig. 2). Thus, tailoring the metamaterial properties, i.e., effective permittivity, can be used to engineer and enhance magneto-optical response in the required spectral range.

Optical second-harmonic generation in reflection from the metamaterial was studied at several angles of incidence for s- and p-polarized fundamental light. It was observed that the SHG radiation is p-polarized and does not reveal azimuthal anisotropy. It was also seen that all SH field is radiated in the direction of the specular reflection. These properties of the SHG response confirm the suitability of the effective medium treatment of the metamaterial for this nonlinear process. Therefore, the considerations of the symmetry of the second-order susceptibility tensor $\chi^{(2)}$ for an in-plane isotropic continuous film can be applied for the studied structure ${ }^{23,24}$, and the SHG response of the metamaterial can be considered as for a nonlinear medium with the dipole axis oriented perpendicularly to the film surface.

Figure 3 shows the SHG intensity spectrum measured when the wavelength of the s-polarized pump beam was scanned in the range 730-830 $\mathrm{nm}$ for a $20^{\circ}$ angle of incidence. A gradual decrease of the SHG intensity is observed with increasing the fundamental wavelength. Similar dependencies were obtained for larger angles of incidence as well as for the p-polarized fundamental beam. In all the cases, the spectra are monotonous and do not reveal any resonant features. Simulations show that the SHG spectra can be described by angular variations of the Fresnel coefficients for the corresponding polarization of the fundamental beam (see inset in Fig. 3) in accordance with the linear reflectivity spectra at the pump and SHG wavelengths shown in Fig. 1 (b).

Figure $4(\mathrm{a}, \mathrm{b})$ shows the spectra of the SHG magnetic contrast $\rho_{2 \omega}$ measured for the p-polarized SHG under the illumination of the sample by s- or p-polarized fundamental light. One can see that for the s-polarized pump beam, a 


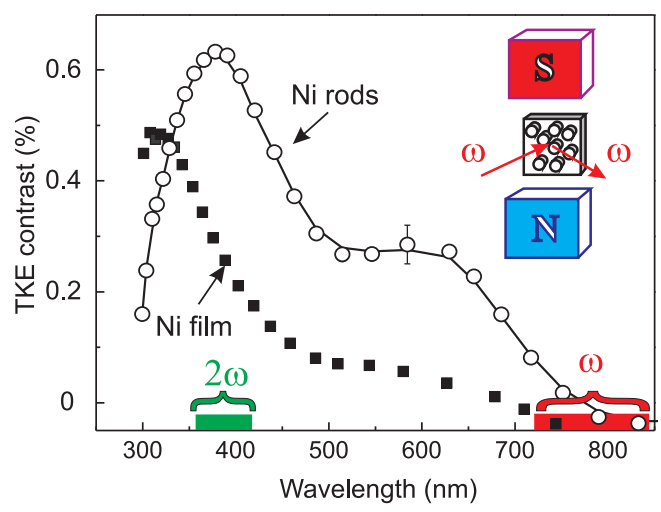

FIG. 2. Experimental spectra of the linear transverse magneto-optical Kerr effect (p-polarized light, angle of incidence $68^{\circ}$ ): (circles) Ni nanorod metamaterial, (squares) continuous Ni film of the same thickness, (line) guide to the eye. Insert shows the experimental geometry.

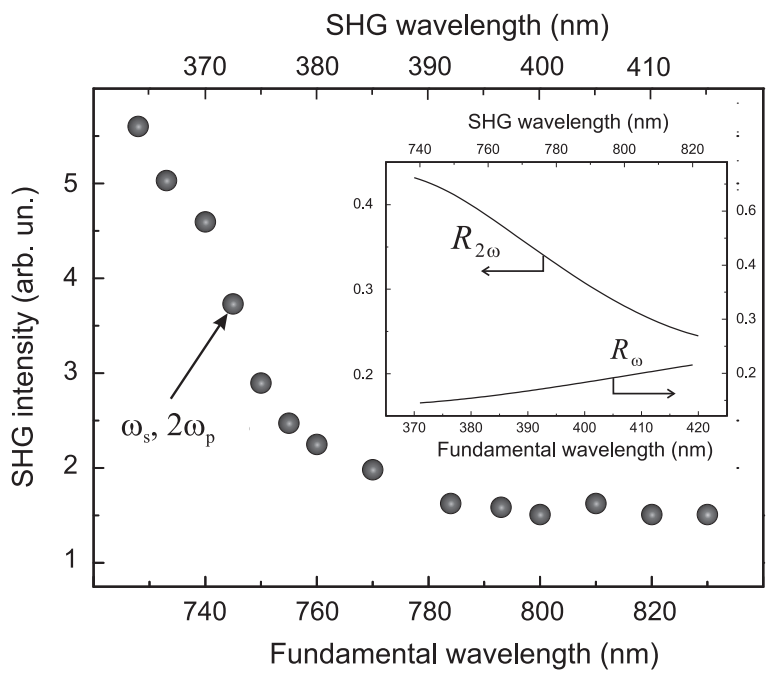

FIG. 3. P-polarized SHG spectra measured at the angle of incidence of $20^{\circ}$ with s-polarized fundamental light. Inset: reflection spectra of the metamaterial at the fundamental $\left(R_{\omega}\right.$, s-polarization) and $\mathrm{SH}\left(R_{2 \omega}\right.$, p-polarization) spectral ranges calculated within the effective medium approximation as in Fig. 1; the angle of incidence is $20^{\circ}$.

strong maximum in the SHG magnetic contrast is observed at about $760 \mathrm{~nm}$ (Fig. 4 (a)) for the angles of incidence of the pump beam of $20^{\circ}$ and of $45^{\circ}$. On the contrary, a monotonous rise of $\rho_{2 \omega}$ is attained at both angles when the wavelength of the p-polarized pump radiation is increased (Fig. 4(b)). Two points are worth noting here: (i) the central wavelength of the SHG contrast maximum for the polarisation combination $\omega_{s}, 2 \omega_{p}$ is twice that for the pronounced maximum in the linear magneto-optical effect (cf. Fig. 2), and (ii) the central wavelength of this maximum does not depend on the angle of incidence of the s-polarized pump beam, so it can not be attributed to the interference effects in the metamaterial layer.

Taking into account the SHG polarization selection rules which follow from the aforementioned anisotropic structure of the metamaterial layer and the attributed symmetry of $\chi^{(2)}$ tensor, it is possible to estimate the contribution of the magnetization-induced SHG processes in the metamaterial. In the case of a magnetic medium, the second-order nonlinear susceptibility has two components, namely, magnetization-induced $\chi^{(2) \text {,magn }}$ and crystallographic $\chi^{(2), c r}$ contributions, each being characterized by its own symmetry. Thus, the SH field is determined by

$$
E_{i}(2 \omega)=E_{i}(2 \omega)^{c r}+E_{i}(2 \omega)^{m a g n}(M) \cos \alpha=\chi_{i j k}^{(2), c r} E_{j}(\omega) E_{k}(\omega)+\chi_{i j k l}^{(2), \operatorname{magn}} E_{j}(\omega) E_{k}(\omega) M_{l}(0) \cos \alpha,
$$

where $\alpha$ is a phase shift between nonmagnetic $\vec{E}_{2 \omega}^{c r}$ and magnetic $\vec{E}_{2 \omega}^{\text {magn }}(M)$ contributions to the SH field and the summation is implied over the reiterative indices. The symmetry analysis shows that in the case of the $\omega_{s} \Rightarrow 2 \omega_{p}$ 


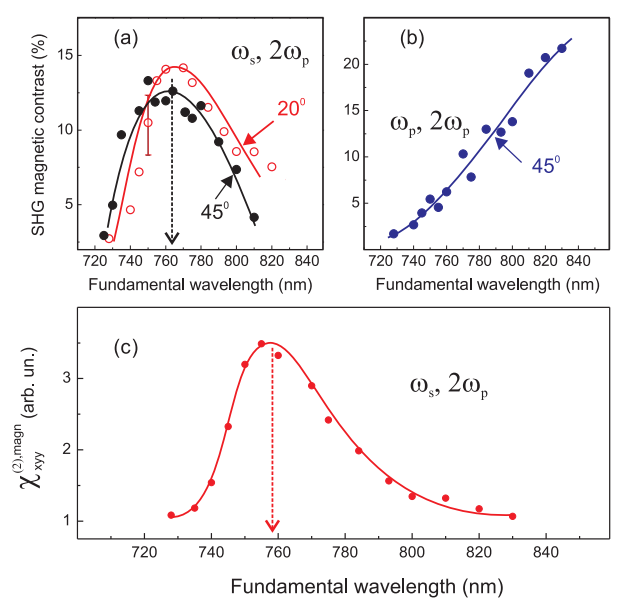

FIG. 4. (a) Spectra of the SHG magnetic contrast measured at $20^{\circ}$ (open circles) and $45^{\circ}$ (filled circles) angles of incidence of the p-polarized SHG under the excitation by s-polarized light; (b) the spectrum of the SHG magnetic contrast measured at the angle of incidence of $20^{\circ}$ for the p-polarized SHG under the excitation with p-polarized light; (c) the spectrum of the secondorder magnetization-induced susceptibility $\chi_{x y y}^{(2), \operatorname{magn}}$ for the s-polarized fundamental light extracted from the experimental data. Lines are guides to the eye.

SHG, there are only two nonzero components, namely, $\chi_{z y y}^{(2), c r}$ and $\chi_{x y y}^{(2), \operatorname{magn} 9}$. In other words, the SH field generated by the s-polarized incident wave $E^{s}(\omega) \vec{e}_{y}$ is

$$
E_{p}(2 \omega)=\chi_{z y y}^{(2), c r} E^{s}(\omega) E^{s}(\omega) \vec{e}_{z}+\chi_{x y y}^{(2), m a g n} E^{s}(\omega) E^{s}(\omega) M_{l}(0) \vec{e}_{x}
$$

where $\vec{e}_{x}, \vec{e}_{z}$ are the unit vectors (see the coordinate frame shown in Fig. 1 (a). While the first term that describes the SHG polarization induced along the $\vec{e}_{z}$ can potentially excite an LSP parallel to the Ni rods' axes, the corresponding frequency range is not accessible in our experiments. At the same time, the second, magnetization-induced, term $\vec{E}_{2 \omega}^{\text {magn }}(M)$ does excite LSP resonance at the SHG wavelength perpendicularly to the nanorod axis. Thus, only magnetization-related SH field can excite the LSP resonance of the nanorods, resulting to much stronger sensitivity of the magnetization-induced SHG to plasmonic properties of nanorods than nonmagnetic SHG.

In order to experimentally extract the spectral dependence of the magnetization-induced component of the SHG susceptibility tensor, $E_{2 \omega}^{\text {magn }}(M) \propto \chi^{(2), \text { magn }}$, one has to know the relative phase shift, $\alpha$, in Eq. 1 . This phase shift can be measured directly using the SHG interferometry technique ${ }^{26}$, which converts the phase-shift between the SH fields generated for the opposite directions of the applied magnetic field into a spatial shift between two interferometric patterns. It was checked experimentally that $\alpha$ is less than $10^{\circ}$ in the spectral range under investigation. Since the SHG magnetic contrast is characterized by the ratio of the magnetization-induced and crystallographic components of the second-order susceptibility tensor: $\rho_{2 \omega} \approx 2 E_{2 \omega}^{\operatorname{magn}}(M) / E_{2 \omega}^{c r} \cos \alpha \propto \chi^{(2), \operatorname{magn}} / \chi^{(2), c r} \cos \alpha$, the spectrum of the effective magnetization-induced susceptibility $\chi^{(2), m a g n}$ was calculated by multiplying the $\rho_{2 \omega}$ by $I_{2 \omega}^{1 / 2}$ and taking into account the measured values of $\cos \alpha$. The corresponding dependence is shown in Fig. 4 (c). It shows a strong nonlinear magneto-optical resonance in s-to-p polarization configuration at around $760 \mathrm{~nm}$ of the fundamental light.

While linear optical and nonlinear-optical properties of the Ni-nanorod metamaterial are well described by the effective medium approximation, the magnetization-induced SHG is specific to individual Ni-nanorods. Due to a weak interaction between the plasmonic modes of the nanorods in the metamaterial, the field enhancement by the LSP associated with individual Ni nanorods seems to play the dominant role. There are no LSP resonances accessible at the spectral range under consideration by the fundamental light, but the LSP mode at $\lambda_{2 \omega}=380 \mathrm{~nm}$ can be excited by the magnetization-induced second-harmonic generation process with the fundamental light at $\lambda_{\omega}=760$ $\mathrm{nm}$. This leads to the observed maximum of the effective magnetic nonlinear susceptibility $\chi^{(2), \text { magn }}$ (Fig. 4 (c)). At the same time, due to the selection rules (Eq. 2), nonmagnetic SHG can not excite these resonances. Thus, magnetization-induced SHG is a sensitive probe for local plasmonic response of the metamaterial.

In conclusion, we have studied both the linear and nonlinear magneto-optical response of Ni nanorod metamaterials. The second-harmonic generation properties confirm the suitability of the metamaterial description of these nanostructures which behave as in-plane homogeneous and isotropic layer. A high sensitivity of magneto-optical and nonlinear magneto-optical Kerr effects have been demonstrated for investigations of weak, localized surface plasmons in the $\mathrm{Ni}$ nanorods. Plasmonic- and metamaterial-related enhancement of the linear and nonlinear magneto-optical response 
has been observed. At the same time, the nonmagnetic optical spectra reveal no resonant features associated with plasmonic excitations of the individual nanorods and are governed by the optical properties of the anisotropic metamaterial layer. The effect is a consequence of the SHG selection rules which allow only magnetization-related SHG to be coupled to transverse LSP of nanorods, resulting in much stronger sensitivity of the magnetization-induced SHG to plasmonic properties of nanorods than non-magnetic SHG. The observed high sensitivity of linear and nonlinear magneto-optical response to the metamaterial photonic modes is promising for tailoring magneto-optical properties, diagnostics of weak plasmonic excitations in magnetic nanostructures and applications in high density data storage. The metamaterial design, e.g., tailoring of effective permittivity with the nanorod geometrical parameters, allows engineering plasmonic and photonic metamaterial modes to further control spectral response of the enhanced magneto-optical effects.

This work was partially supported by EPSRC (UK) and RFBR grants No 10-02-01136, 10-02-00967, by the Federal Program of the Russian Ministry of Education and Science (grant 8393). 
${ }^{1}$ V. Bonanni, S. Bonetti, T. Pakizeh, Z. Pirzadeh, J. Chen, J. Nogus, P. Vavassori, R. Hillenbrand, J. Âkerman, and A. Dmitriev, Nano Lett. 11, 5333 (2011).

2 J.C. Banthi, D. Meneses-Rodriguez, F. Garcia, M.U. González, A. García-Martín, A. Cebollada, G. Armelles, Advanced Materials, 24, OP36 (2012).

3 C. S. Levin, C. Hofmann, T. A. Ali, A. T. Kelly, E. Morosan, P. Nordlander, K. H. Whitmire, N. J. Halas, ACS Nano 3, 1379 (2009).

${ }^{4}$ G. Armelles, A. Cebollada, A. García-Martín, J. M. García-Martín, M.U. González, J B González-Díaz, E Ferreiro-Vila, J.F. Torrado, J. Opt. A: Pure Appl. Opt. 11, 114023 (2009).

5 S. Melle, J.L. Menendez, G. Armelles, D. Navas, M. Vazquez, K. Nielsch, R.B. Wehrspohn, U. Gosele, Appl. Phys. Lett. 83, 4547 (2003).

6 J.B. Gonzalez-Diaz, A. Garcia-Martin, G. Armelles, D. Navas, M. Vazquez, K. Nielsch, R.B. Wehrspohn, U. Gosele, Adv. Mater. 19, 2643 (2007).

7 G. Armelles, J.B. Gonzalez-Diaz, A. Garcia-Martin, Jose M. Garcia-Martin, A. Cebollada, M.U. Gonzalez, S. Acimovic, J. Cesario, R. Quidant, G. Badenes, Optics Express 16, 16104 (2008).

8 J. B. Gonzalez-Diaz, A. Garcia-Martin, J. M. Garcia-Martin, A. Cebollada, G.Armelles, Small 4, 202 (2008).

9 R.-P. Pan, H.D. Wei, Y.R. Shen, Phys. Rev. B 39, 1229 (1989).

10 L. Wang, C. Clavero, Z. Huba, K. J. Carroll, E.E. Carpenter, D. Gu, R.A. Lukaszew, Nano Lett. 11, 1237 (2011).

11 L. Wang, K. Yang, C. Clavero, A.J. Nelson, K.J. Carroll, E.E. Carpenter, R.A. Lukaszew, J. Appl. Phys. 107, 09B303 (2010).

12 G. A. Wurtz, W. Hendren, R. Pollard, R. Atkinson, L. Le Guyader, A. Kirilyuk, Th. Rasing, I. I. Smolyaninov, A. V. Zayats, New J. Phys. 10, 105012 (2008).

13 P. Evans, W. R. Hendren, R. Atkinson, G. A. Wurtz, W. Dickson, A. V. Zayats, R. J. Pollard, Nanotechnology 17, 5746 (2006).

14 A. A. Stashkevich, Y. Roussigne, P. Djemia, S. M. Cherif, P. R. Evans, A. P. Murphy, W. R. Hendren, R. Atkinson, R. J. Pollard, A. V. Zayats, G. Chaboussant, F. Ott, Phys. Rev. B 80, 144406 (2009).

15 Y. Veniaminova, A. A. Stashkevich, Y. Roussign, S. M. Chrif, T. V. Murzina, A. P. Murphy, R. Atkinson, R. J. Pollard, A. V. Zayats, Optical Matetials Express 2, 1260 (2012).

16 A. Wokaun, J.G. Bergman, J.P. Heritage, A.M. Glass, P.F. Liao, D.H. Olson, Phys. Rev. B 24, 849 (1981).

17 O. Aktsipetrov, E. Dubinina, S. Elovikov, E. Mishina, A. Nikulin, N. Novikova, M. Strebkov, Solid State Commun. 70, 1021 (1989).

18 E. Kim, S. Elovikov, T. Murzina, O. Aktsipetrov, M. Bader, G. Marowsky, JETP Lett. 80, 527 (2004).

19 V. K. Valev, A. V. Silhanek, W. Gillijns, Y. Jeyaram, H. Paddubrouskaya, A. Volodin, C. G. Biris, N. C. Panoiu, B. De Clercq, M. Ameloot, O. A. Aktsipetrov, V. V. Moshchalkov, Th. Verbiest, ACS Nano 5, 91 (2011).

${ }^{20}$ G. Bautista, M. J. Huttunen, J. Mäkitalo, J. M. Kontio, J. Simonen, and M. Kauranen, Nano Lett. 12, 3207 (2012)

21 E. Gan'shina, V. Guschin, I.Romanov, A. Tselev, J. Magn. Magn. Mater. 185, 258 (1998).

22 J. Elser, R. Wangberg, V.A. Podolskiy, E.E. Narimanov, Appl. Phys. Lett. 89, 261102 (2006)

23 B. K. Canfield, S. Kujala, K. Jefimovs, Y. Svirko, J. Turunen, M. Kauranen, J. Opt. A: Pure Appl. Opt. 8, S278 (2006).

24 M. Zdanowicz, S. Kujala, H. Husu, M. Kauranen, New Journal of Physics 13, 023025 (2011).

${ }^{25}$ http://www.filmetrics.com/refractive-index-database.

${ }^{26}$ O.A. Aktsipetrov, E.M. Kim, R.V. Kapra, T.V. Murzina, A.F. Kravets, M. Inoue, S.V. Kuznetsova, M.V. Ivanchenko, V.G. Lifshits, Phys. Rev. B, 73, 140404 (2006). 